\title{
Chromosome Study of Yulong Vole (Eothenomys proditor)
}

\author{
Dong-ming Yang ${ }^{1,3}$, Reiqing Liu², Ya-ping Zhang ${ }^{1}$, \\ Zhiping Chen ${ }^{3}$ and Yingxiang Wang ${ }^{3}$

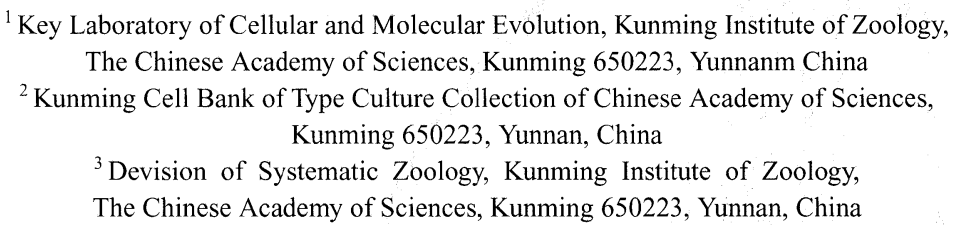

Accepted September 2, 1998

\begin{abstract}
Summary A first description of the karyotype of Yulong vole (Eothenomys proditor) is presented. Karyotype studies were carried out using conventional staining, G-banding, C-banding and silverstaining. Yulong vole was sharp different from other allied voles on karyotype. The diploid number is $2 n=32$, with the fundamental number of 56 . The autosomes consist of 13 pairs of meta-/submetacentric elements and two pairs of arcrocentric elements. The $\mathrm{X}$ chromosome is medium sized and submetacentric, whereas the $\mathrm{Y}$ chromosome is small and subacrocentric. The G-banding allows to identify chromosome pairing and to detect the chromosome rearrangements intervening in karyotypic differentiation. In the C-banded karyotype, the centromeric heterochromatin is observed in Nos. 2, 4 and 12 chromosomes, the terminal C-band is found in No. 13 and X chromosomes. Y chromosome is completely C-band-positive, moreover, the interstial distribution of C-bands has been demonstrated on the biarm of No. 2 chromosome pair and the long arm of Nos. 2, 9 and 10 chromosomes respectively. Nucleolus organizer regions (NORs) appeared in the long arm of Nos. 1, 2 and 4 chromosomes and short arm of No. 11 chromosome.
\end{abstract}

The genus Eothenomys is mainly distributed in Transhimalayan mountains, the southwestern China. Based mainly on morphology, from 9-13 nominal forms have been assigned to the genus which includes 2-3 subgenera (Allen 1940, Ellerman and Morrison-Scott 1951, Corbet 1978, Nowak and Paradiso 1983, Corbet and Hill 1991). However, the taxonomic status of most of these species is still confusing and need of review. Yulong vole (Eothenomys prodior) is an endemic specie living in Lijiang region, Yunnan province of China. It was originally named as a new specie of Eothenomys by Hinton (1923). Allen (1940) changed it under Anteliomys, and his point has been acknowledged by most taxonomists. Nevertheless, the status of Anteliomys is disputable. Cytogenetics is a powerful tool to investigate classification and evolution. However, the chromosome data of Eothenomys was scant. Until now, only E. melanogaster and E. miletus have been karyotyped by conventional and/or differential staining (Wu et al. 1989, Chen et al. 1994, Harada et al. 1991). In this paper, the conventional and banded karyotypes of Yulong vole (E. prodior) were first described, and we preliminarily discussed the karyotype evolution and taxonomic situation of this specie. We hope this study can provide new information about phylogeny of this genus and its related genera.

Materials and methods

Four animals (two females, two males) were captured alive in Yulong snow mountain, Northern Yunnan province of China.

The chromosomes studied were from fibroblast cells which were obtained from primary lung tissue culture by means of standard air-drying methods. For chromosomal classification, the method 
of Leaven et al. (1964) was adapted. The procedures for G-banding was the same as the trypsin digestive method of Seabright (1971). The C-banding was produced by a slight modification of Sumner's technique (1972). For the detection of Ag-NORs, the slides were stained with silver (Howell and Black 1980).

\section{Result}

The diploid chromosome number of Yulong vole was found to be $32(2 n=32)$ based on the data from a total of 100 metaphases, and the fundamental arm number is $54(\mathrm{FN}=56)$. The autosomoses of Yulong vole consisted 7 metacentric pairs (M), 6 submetacentric pairs (SM) and 2 acrocentric pairs (A). The $\mathrm{X}$ chromosome was a medium $\mathrm{M}$ element while the $\mathrm{Y}$ chromosome was a small A element (Fig. 1A). So the karyotype formula of Yulong vole was 32, XY(M, A)+ $14 \mathrm{M}+12 \mathrm{SM}+4 \mathrm{~A}$ (Fig. 1A, Table 1).

The G-bands of the most autosomoses and X chromosome were-segmented, but Nos. 15 and $\mathrm{Y}$ chromosomes were indistinct (Fig. 1B). Based on the G-band pattern, we can identify the homologous chromosome pairs which are equivocal in conventional karyotype, and demonstrate the similarity of karyotype in different species.

The Yulong vole contains a small amount of heterochromatin (Fig. 1C). The pericentromeric regions of Nos. 2, 4 and 12 chromosomes; the terminal of short arm of $\mathrm{X}$ and No. 13 chromosome pair, and the entire length of $Y$ chromosome were all stained positively. Moreover we found interstitial heterochromatin on Nos. 2, 9 and 10 chromosomes, however, it was very faint on the long arms of Nos. 2 and 12 chromosomes (Fig. 1C, shown by arrows). Slight C-bands were also found on the two terminals of No. 1 chromosomes (not shown).

Silver-staining karyotype is shown in Fig. 1D. Four pairs of Ag-NORs were observed, and they

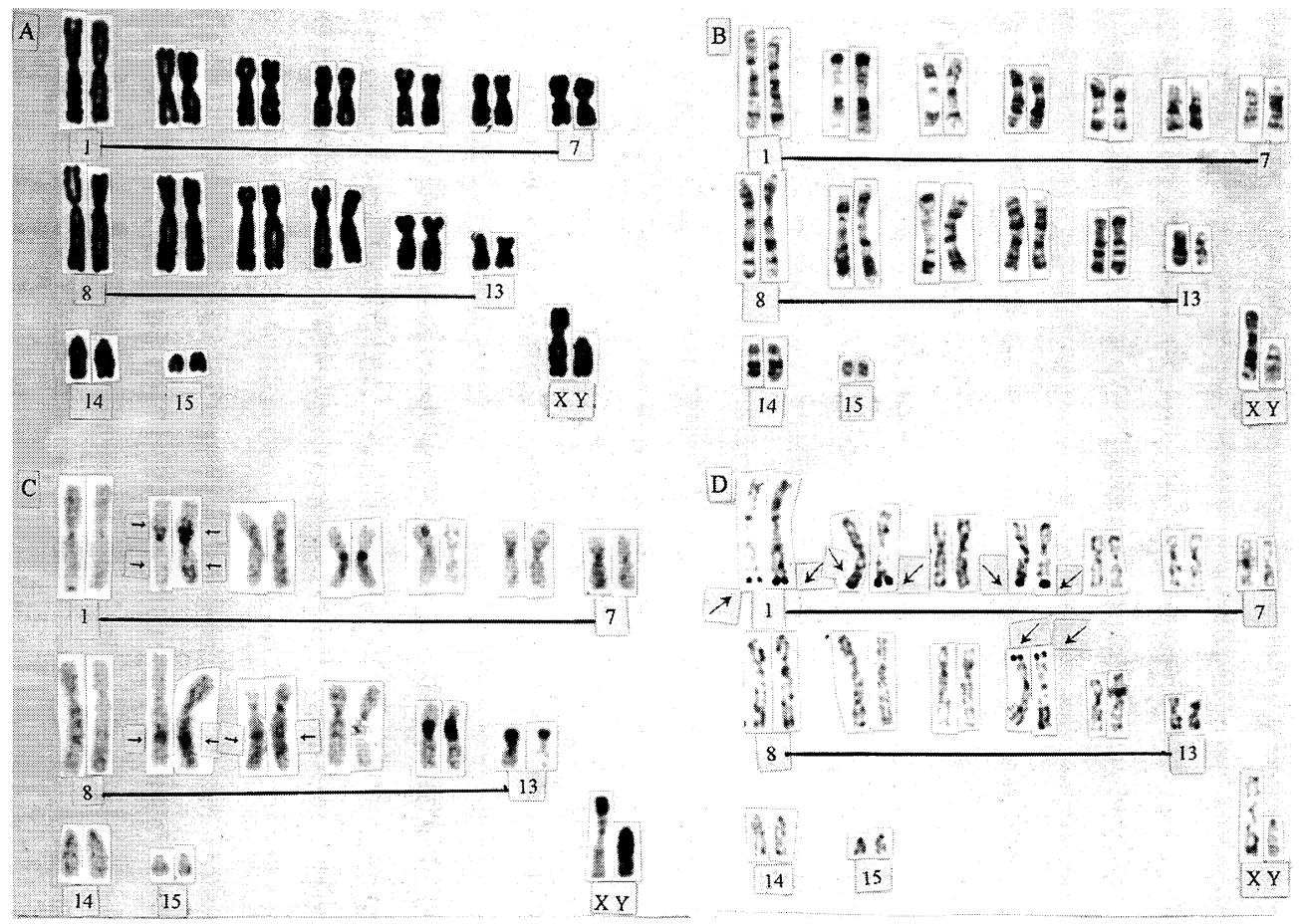

Fig. 1. A) The conventional karyotype of Yulong vole. B) The G-banded karyotype of Yulong vole. C) The C-banded karyotype of Yulong vole (arrows show the interstitial heterochromatin). D) The silver stained karyotype of Yulong vole (arrows show the NORs). 
Table 1. The measurement of the chromosomes of Yulong vole

\begin{tabular}{|c|c|c|c|c|}
\hline Nos. & Position of centromere & Length & Relative length & Arm ratio \\
\hline 1 & M & $13.94 \pm 0.22$ & $10.35 \pm 0.16$ & 1.25 \\
\hline 2 & M & $10.22 \pm 0.42$ & $7.57 \pm 0.37$ & 1.09 \\
\hline 3 & M & $9.85 \pm 0.29$ & $7.32 \pm 0.18$ & 1.59 \\
\hline 4 & M & $8.37 \pm 0.32$ & $6.21 \pm 0.22$ & 1.21 \\
\hline 5 & M & $7.13 \pm 0.15$ & $5.39 \pm 0.11$ & 1.23 \\
\hline 6 & M & $7.16 \pm 0.20$ & $5.30 \pm 0.17$ & 1.59 \\
\hline 7 & M & $6.95 \pm 0.18$ & $5.09 \pm 0.13$ & 1.27 \\
\hline 8 & SM & $14.13 \pm 0.39$ & $10.51 \pm 0.23$ & 2.22 \\
\hline 9 & SM & $12.77 \pm 0.26$ & $9.49 \pm 0.15$ & 1.73 \\
\hline 10 & SM & $11.36 \pm 0.42$ & $8.84 \pm 0.35$ & 2.12 \\
\hline 11 & SM & $10.46 \pm 0.31$ & $7.79 \pm 0.19$ & 1.89 \\
\hline 12 & SM & $8.44 \pm 0.14$ & $6.28 \pm 0.11$ & 1.88 \\
\hline 13 & SM & $5.19 \pm 0.26$ & $3.85 \pm 0.21$ & 1.72 \\
\hline 14 & A & $5.92 \pm 0.08$ & $4.40 \pm 0.05$ & - \\
\hline 15 & A & $2.69 \pm 0.05$ & $2.00 \pm 0.03$ & - \\
\hline $\mathrm{X}$ & M & $10.18 \pm 0.35$ & $7.51 \pm 0.26$ & 1.52 \\
\hline $\mathrm{Y}$ & $\mathrm{A}$ & $5.52 \pm 0.12$ & $4.13 \pm 0.07$ & - \\
\hline
\end{tabular}

$\mathrm{M}=$ metacentric, $\mathrm{SM}=$ submetacentric, $\mathrm{A}=$ acrocentric.

are located in the terminal of Nos. 1, 2, 4 and 11 chromosome pairs respectively (Fig. 1D, shown by arrows). Also, we observed associations of Ag-NORs, however, the frequency of association was very low (about $2 \%$ ).

\section{Discussion}

Genera Eothenomys, Clethrionomys and Caryomys are similar with each other on the basis of morphologic character, so they are placed in the tribe Clethrionomyini of subfamily Arvicolinae (Koenigswald 1980). Also karyotypes in the three genera reported are similar with each other. As show in Table 2, the karyotypes of all specie are 2n=56 (except Caryomys inez and C. eva), and include 1 pair of smaller metacentric chromosomes (except Eothenomys miletus and E. eleusis). $\mathrm{FN}=54-60$, variation in FN was very small. In this paper, the karyotype of Yulong vole was first reported, and the result showed that Yulong vole cytogenetically differed sharply from other voles of the tribe Clethrionomyini. The cytogenetic findings suggest that Yulong vole takes an important role in the classification and phylogeny of the tribe Clethrionomyini.

Carleton and Myeris (1979) stated that, in a group of given taxon, a primitive karyotype is most likely with a high $2 \mathrm{n}$ consisted of mostly acrocentrics while a derived one with a less $2 \mathrm{n}$ consisted of mostly biarmed chromosomes. The diploid number of Yulong vole is 32, and most chromosomes are biarmed (Fig. 1A, Table 1). According to above point, Yulong vole is a relative derived specie in the tribe Clethrionomyini. Heterochromatin is a genetic structure that may facilitate chromosome rearrangement. Usually, the ancestral C-banded karyotype have two features. (1) all autosomes and $\mathrm{X}$ chromosome carried $\mathrm{C}$-bands and the centromeric heterochromatin was abundant; (2) Y chromosome was completely heterochromtic (Sen and Sharma 1983). It is obvious from Fig. $1 \mathrm{C}$ that the elements carrying centromeric heterochromatin decreased in the complement of Yulong vole; while the elements carrying terminal heterochromatin and interstitial heterochromatin increased. Moreover, the terminal heterochromatin is demonstrated on the short arm of $\mathrm{X}$ chromosomes, this uncommon distribution of $\mathrm{C}$-band had been found in quite a few differed species, such as Rattus rattus, Tragulus javanicus, Martes foina, Marts flavigula, Hylobates hoolock, Hylobates leucogenys and others (Yosida and Sagai 1975, Shi and Chen 1989, Liu et al. 1994, Chen et al. 
Table 2. Karyotype comparison among Yulong vole and species of Clethrinomys, Caryomys, Phaulomys and Eothenomys

\begin{tabular}{|c|c|c|c|c|}
\hline Species & $2 n$ & $\mathrm{FN}$ & Chromosome formula & References \\
\hline C. rutilus & 56 & 56 & $\mathrm{XY}(\mathrm{A}, \mathrm{A})+2 \mathrm{M}+52 \mathrm{~A}$ & Kral 1971 \\
\hline C. gapperi & 56 & $\begin{array}{c}56 \\
\text { or } 58\end{array}$ & $\begin{array}{c}\mathrm{XY}(\mathrm{A}, \mathrm{A})+2 \mathrm{M}+52 \mathrm{~A} \\
\text { or } \mathrm{XY}(\mathrm{A}, \mathrm{SA})+2 \mathrm{M}+2 \mathrm{SA}+50 \mathrm{~A}\end{array}$ & $\begin{array}{l}\text { Hsu et al. } 1970 \\
\text { Frechette } \text { et al. } 1971\end{array}$ \\
\hline C. glareolus & 56 & 56 & $X Y(A, M)+2 M+52 A$ & Hsu et al. 1970, Kuliev et al. 1977 \\
\hline C. imaizuni & 56 & 56 & $\mathrm{XY}(\mathrm{SA}, \mathrm{SA})+2 \mathrm{M}+52 \mathrm{~A}$ & Mattey 1973 \\
\hline C. niigatae & 56 & 56 & $\mathrm{XY}(\mathrm{A}, \mathrm{M})+2 \mathrm{M}+52 \mathrm{~A}$ & Hsu et al. 1974 \\
\hline C. rufocanus & 56 & 56 & $\mathrm{XY}(\mathrm{A}, \mathrm{A})+2 \mathrm{M}+52 \mathrm{~A}$ & Hsu et al. 1969 \\
\hline C. r. shaneius & 56 & 56 & $\mathrm{XY}(\mathrm{M}, \mathrm{A})+2 \mathrm{M}+52 \mathrm{~A}$ & Jiang et al. 1991 \\
\hline C. rutilus & 56 & 56 & $\mathrm{XY}(\mathrm{A}, \mathrm{A})+2 \mathrm{M}+52 \mathrm{~A}$ & Kral 1971 \\
\hline C. frater & 56 & 56 & $\mathrm{XY}(\mathrm{A}, \mathrm{A})+2 \mathrm{M}+52 \mathrm{~A}$ & Jiang et al. 1993 \\
\hline Car. inez & 54 & 60 & $\mathrm{XY}(\mathrm{SM}, \mathrm{A})+4 \mathrm{M}+4 \mathrm{SA}+44 \mathrm{~A}$ & Wang et al. 1991 \\
\hline Car: eva & 54 & 56 & $\mathrm{XY}(\mathrm{M}, \mathrm{A})+2 \mathrm{M}+2 \mathrm{SA}+48 \mathrm{~A}$ & Ma et al. 1996 \\
\hline Pha. kageus & 56 & $\begin{array}{l}56 \\
60\end{array}$ & $\begin{array}{c}\mathrm{XY}(\mathrm{SM}, \mathrm{A})+2 \mathrm{M}+52 \mathrm{~A} \\
\text { or } \mathrm{XY}(\mathrm{SA}, \mathrm{SA})+2 \mathrm{M}+4 \mathrm{SA}+48 \mathrm{~A}\end{array}$ & $\begin{array}{l}\text { Hsu et al. } 1974 \text {, } \\
\text { Ando et al. } 1988\end{array}$ \\
\hline Pha. smithi & 56 & 56 & $\begin{array}{c}\mathrm{XY}(\mathrm{A}, \mathrm{A})+2 \mathrm{M}+52 \mathrm{~A} \\
\text { or } \mathrm{XY}(\mathrm{SA}, \mathrm{SA})+2 \mathrm{M}+4 \mathrm{SA}+48 \mathrm{~A}\end{array}$ & $\begin{array}{l}\text { Hsu et al. } 1974, \\
\text { Ando et al. } 1988\end{array}$ \\
\hline E. miletus & 56 & 54 & $\mathrm{XY}(\mathrm{A}, \mathrm{A})+54 \mathrm{~A}$ & Wu et al. 1989, Chen et al. 1994 \\
\hline E. melanogaster & 56 & 56 & $\mathrm{XY}(\mathrm{A}, \mathrm{A})+2 \mathrm{M}+52 \mathrm{~A}$ & Harada et al. 1991 \\
\hline E. eleusis & 56 & 54 & $\mathrm{XY}(\mathrm{A}, \mathrm{A})+54 \mathrm{~A}$ & Yang Unpublished \\
\hline E. proditor & 32 & 56 & $\mathrm{XY}(\mathrm{SM}, \mathrm{A})+14 \mathrm{M}+12 \mathrm{SM}+4 \mathrm{~A}$ & Present paper \\
\hline
\end{tabular}

C. $=$ Clethrinomys, Car $=$ Caryomys, $E .=$ Eothenomys, Pha. $=$ Phaulomys, $\mathrm{M}=$ metacentric chromosome, $\mathrm{SM}=$ submetacentric chromosome, $\mathrm{SA}=$ subacrocentric chromosome, $\mathrm{A}=$ acrocentric chromosome.

1990, Liu et al. 1996, Hsu 1978). Hsu et al. (1971) interpreted that the appearing of terminal heterochromatin and interstitial heterochromatin is result of pericentric inversions and asymmetrical translocations, and they thought it of one sign of differentiation. Data above-mentioned suggest that Yulong vole might be more differed than other voles of tribe Clethrionomyini.

The chromosome rearrangement is a primary event in initiating divergence of speciation (White 1978). Yosida (1973) stated that the frequency of chromosome evolution is high in rodents, however, it differs by species. In the genus Eothenomys, the karyotype evolution of E. miletus and E. eleusis is slow while of Yulong vole is fast. The reason may be that the former two with 56 acrocentric chromosomes are primitive and from them karyotype evolution occurred. Fossil evidence also showed that Anteliomys fauna occurs later than Eothenomys fauna (Zheng 1993). For the mode and direction of chromosomal evolution in rodents, Nadler (1969) proposed the fusion hypothesis that centric fusion is the commonest mechanism of structural rearrangement. Most species of the tribe Clethrionomyini have diploid number that vary between 54-56 with FN between 54-60. Although the diploid number of Yulong vole is far from the lower limit of the variation range for this character in those genera, FN is just in the variation range (Table 2). This drastic reduction in the numbers of $2 \mathrm{n}$ without affecting the $\mathrm{FN}$ suggests that chromosomal differentiation in Yulong vole has occurred basically through centric fusion. However, comparing chromosomes of Yulong vole and other Eothenomys known for G-banded karyotype, we found Yulong vole shares little similarities with other Eothenomys (Yang, unpublished). This indicates that Robertson fusion is not sufficient to explain the karyotype evolution in here. Several other structural rearrangements have also contributed to the great chromosomal divergence that appeared in Yulong vole karyotype. Considering chromosome morphology, G-banding patterns and C-banding patterns together, we infer that the Yulong vole originated from a E. miletus-like ancestor, and the karyotype evolutionary way of Yulong vole was not only Robertsonian fusion but also tandem translocation and pericentric inversion. The ancestor of Yulong vole might has 28 pairs of chromosomes, which were all A elements with centromeric heterochromatin. Under the press of selection, two acrocentric autosomes fused in 
the centromeric areas to form a biarmed element; the formation of biarmed autosomes with terminal or interstitial heterochromatin involved in centromere-telomere translocations. Also, changes in the amount of heterochromatin have occurred in the karyotypic evolution. For instants, heterochromatic arm of No. 13 chromosome pair seems to be caused by addition of constitutive heterochromatin, and the presence of terminal heterochromatin in X chromosome may be the result of asymmetrical translocation.

According to Allen's opinion, the genus Eothenomys includes three subgenera: Eothenomys, Anteliomys and Caryomys. However the status of Anteliomys and Caryomys is still in discussion some taxonomists regarded it as an independent genus (Hinton 1926, Repenning et al. 1990, Gromov and Polyakov 1992), others thought of it as a subgenus of Eothenomys (Ellerman and Morrison-Scott 1951, Corbet 1978, Musser et al. 1993). Ma and Jiang (1996) found that the morphological and karyotype characteristics of the two species from Caryomys was distinct from Eothenomys and Clethrinomys, so they reinstated the genus Caryomys. At present most taxonomist acknowledged putting Yulong vole under the Anteliomy. However, when the skull morphology, dental morphology, comparative karyology and fossil evidence were considered, Yulong vole should be placed in a derived category. Whether separated Yulong vole from Eothenomys or refund the genus Anteliomys is still an open question because hitherto Karyological information on Anteliomys is extremely scanty and there has not been conclusive data. Further studies of other voles of Anteliomys is now in progress, and will be reported in the future.

\section{Acknowledgment}

We are very grateful to Mr. Sou Lin for his help on capturing animals. Our sincere thanks also due to Mrs. Yuze Chen, Mrs. Jinhuan Wang and Mrs. Wenhui Ni for their technical assistance, and to Dr. Xuelong Jiang for language revision of the English manuscript. This work was gone ahead in the Kunming Cell Bank of Type Culture of Chinese Academy of Sciences. It is supported by the Natural Science Foundation of China, the Applied Basic Research Foundation of Yunnan and Laboratory of Cellular and Molecular Evolution Kunming Institute of Zoology, The Chinese Academy of Sciences.

\section{References}

Allen, G. M. 1940. Mammals of China and Mongolia. Amer. Mus. Nat. Hist., New York. Part II: 820-823.

Ando, A., Shiraishi, S., Harada, M. and Uchida, T. A. 1988. A karyological study of two intraspecific taxa in Japanese Eothenomys (Mammalia: Rodentia). J. Mammal. Soc. Japan. 13 (2): 93-104.

Carleton, M. D. and Myers, P. 1979. Karyotypes of some harvest mice, genus Reithrodontomys. J. Mamm. 60 (2): $307-313$.

Chen, Z. P., Jiang, X. L. and Wang, Y. X. 1994. Studies on the karyotype of Oriental vole (Eothenomys miletus). Cytologia. 59: 289-293.

-, Liu, R. Q. and Wang, Y. X. 1996. Chromosome study of yellow-throated marten (Martes flavigula). Acta Theriologica Sinica (in Chinese) 10(1): 19-22.

Corbet, G. B. 1978. The Mammals of the Palaearctic Region: A Taxonomic Review. Brit. Mus. Nat. Hist., Cornell Univ. press, London \& Ithaca. 101.

Ellerman, J. R. and Morrison-Scott, T. C. S. 1951. Checlist of Palaearctic and Indian Mammals. Brit. Mus. Nat. Hist., London. 667-669.

Frechette, A. G. and Jalal, S. M. 1971. Karyological study of two subspecies C. gapperi gapperi, C. g. Loringi of the redblacked vole. Mammal. Chrom. News. 12: 38 .

Gromov, I. M. and Polyakov, I. Y. 1992. Fauna SSSR, Mlekopitayushchie, tom 3, vyp. 8 [Fauna of USSR, vol. 3, pt. 8, mammals]. Polevki [voles (microtinae)]. E. J. Brill. Leiden-New York-København-Koln. 504.

Harada, M., Ando, A., Lin, L. K. and Takada, S. 1991. Karyotypes of the Taiwan Vole Microtus kikuchii and the Pere David's Vole Eothenomys melanogaster from Taiwan. J. Mammal. Soc. Japan. 16 (1): 41-45.

Hinton, M. A. C. 1926. Monograp of the Voles and lemming (Microtinae), Living and Extinct. Brit. Mus. Nat. Hist., London. 291 . 
Howell, W. M. and Black, D. A. 1980. Controlled silver-staining of nucleolus organizer regions with a protective colloidal developer: A 1-step method. Experientia 36: 1014-1015.

Hsu, T. C. 1978. Human and Mammalian Cytogenetics: An Historical Perspective. Springer-Verlay.

- and Arrighi, F. E. 1975. Distribution of constitutive heterochromatin in mammalian chromosomes. Chromosoma 53: $25-26$.

- and Benirschke, K. 1969. An Atlas of Mammalian Chromosomes. vol. 3. Sprinter, New York.

— and - 1970. An Atlas of Mammalian Chromosomes. vol. 4. Sprinter, New York.

— and - 1974. An Atlas of Mammalian Chromosomes. vol. 8. Sprinter, New York.

Koenigswald, W. V. 1980. Schmelzstruktur und Morphologie in den Molaren der Arvicolidae (Rodentia). Abhandlungen der Senckenbergischen Naturforschenden. Gesellschaft 539: 1-129.

Kral, B. 1971. Chromosome characteristics of Murridae and Microtidae from Czechoslovakia. Acta Sci. Brno 6: 1-78.

Leaven, A., Fredga, K. and Sinderg, A. A. 1964. Nomenclature for centromeric position on chromosome. Hereditas 52 (2): 201-220.

Liu, R. Q., Nie, W. H. and Chen, Y. Z. 1994. A stone marten with diploid chromosome number of 38. Chrom. Infor. Serv. 56: 15-16.

- - - - - and Yu, D. H. 1993. Comparative studies of C-band and Ag-NORs on the chromosomes between two species gibbon. Acta Theriologica Sinica (in Chinese) 16 (3): 182-1871.

Ma, Y. and Jiang, J. Q. 1996. The reinstatement of the status of genus Caryomys (Thomas 1911) (Rodentia: Microtinae). Acta Zootaxonomica Sinica (in Chinese) 21 (4): 493-497.

Musser, G. G. and Carleton, M. D. 1993. Family Microtus. In: Wilson, D. E. and Reeder, D. A. M. (eds.) Mammal Species of the World. A Taxonomic and Geographic Reference. Smiths. Inst., Washington and London.

Nadler, C. F. 1969. Chromosomal evolution in rodents. In: Benirschke, K. (ed.) Comparative Mammalian Cytogenetics. Springer-Verlag, Berlin. Heidelberg. New York. 227-309.

Seabright, M. 1971. A rapid banding technique for human chromosomes. Lancet 2: 971-972.

Sen, S. and Sharma, T. 1983. Role of constitutive heterochromatin in evolutionnary divergence: results of chromosome banding and condensation inhibition studies in Mus mus musculus, Mus booduga and Mus dunni. Evolution 37: 628-636.

Shi, L. M. and Chen, Y. Z. 1989. The karyotype analysis of Yunnan mouse deer (Tragulus javanicus williamsoni). Acta zoologica Sinica (in Chinese) 35 (1): 41-44.

Sumner, A. T. 1972. A simple technique for demonstrating centromeric heterochromatin. Exp. Cell Res. 75: 304-306.

White, M. J. D. 1978. Modles of Speciation. W. H. Freeman and Company, San Francisco.

Wu, A. G., Wang, G. L. and Zhao, H. 1989. The karyotype of Eothenomys miletus. Acta Theriologica Sinica 9 (3): 231.

Yosida, T. H. 1973. Karyotype and evolution. Iden (The Heredity) (in Japanese) 27 (7): 60-66.

- and Sagai, T. 1975. Variation of C-band in the chromosomes of several subspecies of Rattus rattus. Chromosoma 50: 283-300.

Zheng, S. H. 1993. Quatemary Rodents of Sichan-Guizhou Area, China. Science Press, Academia Sinica. 219-220. 